\title{
General Dynamical Equations of Motion for Elastic Body Systems
}

\author{
Shui-Lin Weng* and Donald T. Greenwood $\dagger$ \\ University of Michigan, Ann Arbor, Michigan 48109
}

\begin{abstract}
A modeling technique capable of determining the time response of a single body (rigid or flexible) that is, in general, undergoing large elastic deformations, coupled with large, nonsteady translational and rotational motions, is presented. The derivations of the governing equations of motion are based on Lagrange's form of d'Alembert's principle. The general dynamical equations of motion are expressed in terms of stress and strain tensors, kinematic variables, the velocity and angular velocity coefficients, and generalized forces. The formulation of these equations is discussed in detail. Numerical simulations that involve finite elastic deformations coupled with large, nonsteady rotational motions are presented for a beam attached to a rotating base. Effects such as centrifugal stiffening and softening, membrane strain effect, and vibrations induced by Coriolis forces are accommodated. The effects of rotary inertia as well as shear deformation are also included in the equations of motion. Although discussions here are restricted to a single body, the formulation allows the capability of a general dynamical formalism for handling multibody (rigid or flexible) dynamics.
\end{abstract}

\section{Introduction}

$\mathbf{H}$ OOKER and Margulies ${ }^{1}$ as well as Roberson and Wittenburg $^{2}$ wrote early papers in the mid-1960s that dealt primarily with spacecraft dynamics. The methodologies they presented were applicable only to systems of rigid bodies with simple joints in a restricted configuration. Later, a number of studies based on these methods were published. ${ }^{3,4}$ A typical modern spacecraft consists of structural subsystems, some essentially rigid and others extremely flexible, frequently interconnected in a time-varying manner. Formulation dealing with this category of spacecraft is a procedure that employs discrete coordinates to describe the unrestricted motions of those structural subsystems idealized as rigid bodies, in combination with distributed or modal coordinates to describe the timevarying deformations of those structural subsystems idealized as flexible elastic appendages. ${ }^{5,6}$ Advances also have been made concerning the coupling effects between gross translational or rotational motion and the elastic deformation of elastic bodies. ${ }^{7-11}$

Repeated numerical simulations are needed to establish a satisfactory design for the prototype of a spacecraft. In an effort to facilitate the simulations of multiple interconnected bodies, analysts have come to rely more and more on general multibody dynamics formalisms. A dynamical system may be translating or spinning in whole or in part and may be expected to undergo large changes in inertial position and orientation. It has become necessary to devise methods of dynamic analysis that combine the generalities of nonlinearities and large motions with the computational efficiency afforded by the use of modal coordinates in describing the vehicle deformations.

Mathematical modeling tools are used to analyze the problems and to derive the dynamical equations of motion for a multibody system. Comparative studies ${ }^{12}$ suggest that Kane's method $^{13,14}$ or some related generalization of Lagrange's form of d'Alembert's principle ${ }^{15}$ most closely combines the two computational advantages: 1) the nonworking constraint forces

Received Nov. 15, 1990; revision received Nov. 15, 1991; accepted for publication Nov. 27, 1991. Copyright (C) 1992 by the American Institute of Aeronautics and Astronautics, Inc. All rights reserved.

*Graduate Research Assistant, Department of Aerospace Engineering; currently, Researcher, Center for Aviation and Space Technology, ITRI, Hsinchu, Taiwan. Member AIAA.

†Professor, Department of Aerospace Engineering. Associate Fellow AIAA. and torques do not appear and 2) the resulting equation set is of minimum dimension.

A modeling technique capable of determining the time response of a rigid or flexible body that is, in general, undergoing large elastic deformations, coupled with large, nonsteady translational and rotational motions, is considered. The derivations of the governing equations of motion are based on Lagrange's form of d'Alembert's principle. The general dynamical equations of motion are expressed in terms of stress and strain tensors, kinematic variables, the velocity and angular velocity coefficients, and generalized forces. These equations can be derived systematically.

It is well known that when flexible structural elements are attached to a rotating base, the apparent stiffness of the structural elements varies with the magnitude of the inertial angular velocity of the spinning base. Also, in linear structural theory, the transverse vibration of a beam is calculated without considering axial forces. But in some cases, e.g., in rapidly rotating turbine or helicopter blades, it is not possible to ignore the effect of axial forces on the bending vibration of blades. When the beamlike blade is spinning, so-called centrifugal stiffening effects that are due to the presence of axial (centrifugal) forces come into play. Coupling betwen centrifugal forces and bending moments makes a rapidly spinning beam stiffer than is predicted by linear theory. In the light of this situation, it is important that a multibody formulation correctly reflects motion-induced stiffness.

To resolve the difficulties, a single generalized formalism, the general dynamical equations of motion, is introduced. It is distinguished from a method using the shortening effect ${ }^{16-18}$ explicitly in that here the centrifugal stiffening terms enter the final equations through the potential energy rather than through the kinetic energy. Also, we investigate the mechanism of motion-induced stiffness variations in various types of elastodynamic structures undergoing large overall motions. Effects such as centrifugal stiffening and softening, membrane strain effect, and vibrations induced by Coriolis forces are accommodated. The effects of rotary inertia as well as shear deformation are also included in the equations of motion.

In Sec. II, we include the finite displacement theory of elasticity, the principle of virtual work, and the preliminaries to the actual dynamical problem for the purpose of completeness. In Sec. III, a generalized formalism to analyze the dynamical system, the general dynamical equations of motion based on Lagrange's form of d'Alembert's principle, is developed. In Sec. IV, an analysis of a single flexible beam is pre- 
sented to illustrate the formulating procedures of the general dynamical equations of motion. Section $\mathrm{V}$ applies the flexible beam theory of Sec. IV to several problems. Section VI presents the conclusions.

\section{Modeling of Flexible Bodies}

In this investigation, we assume that a rigid or deformable body may experience large translational or rotational displacements relative to an inertial coordinate system. Although a body-axis system that is rigidly attached to a point on the body is commonly employed as a reference for rigid components, there are many arrangements for the body axes of flexible components. ${ }^{19,20}$ The origin of this reference frame does not have to be rigidly attached to a point on the deformable body, but frequently it is so chosen. We will attach the body axes to a small rigid volume element at one end of the beam.

\section{Analysis of Strain}

In the present section, we shall treat the finite displacement theory of elasticity in rectangular Cartesian coordinates and employ the Lagrangian approach, in which the coordinates defining a point of the body before deformation are employed for locating the point during the subsequent deformation.

Let a rectangular Cartesian coordinate system $x^{1} x^{2} x^{3}$ be assigned to each body, and let the relative position vector of an arbitrary point $P^{(0)}$ of the body before deformation be represented by

$$
r^{(0)}=r^{(0)}\left(x^{1}, x^{2}, x^{3}\right)
$$

as shown in Fig. 1, where the superscript (0) means that the quantity refers to the state before deformation. The base vectors in this coordinate system are given by

$$
\boldsymbol{j}_{\mu}=\frac{\partial \boldsymbol{r}^{(0)}}{\partial x^{\mu}}=\boldsymbol{r}_{, \mu}^{(0)}, \quad(\mu=1,2,3)
$$

where the notation ( $)_{, \mu}$ denotes differentiation with respect to $x^{\mu}$. The base vectors are unit vectors in the direction of the coordinate axes and are mutually orthogonal.

The body is now assumed to be deformed into a strained configuration. The points $P^{(0)}, Q^{(0)}, R^{(0)}, S^{(0)}$, and $T^{(0)}$ move to new positions denoted by $P, Q, R, S$, and $T$, respectively, and the infinitesimal rectangular parallelepiped is deformed into a parallelepiped that, in general, is no longer rectangular. Let us denote the position vector of the point $P$ relative to the body frame by

$$
r=r\left(x^{1}, x^{2}, x^{3}\right)
$$

where $\left(x^{1}, x^{2}, x^{3}\right)$ are actually the Cartesian coordinates of $P^{(0)}$. Introduce the lattice vectors defined by

$$
L_{\mu}=\frac{\partial r}{\partial x^{\mu}}=r_{, \mu}, \quad(\mu=1,2,3)
$$

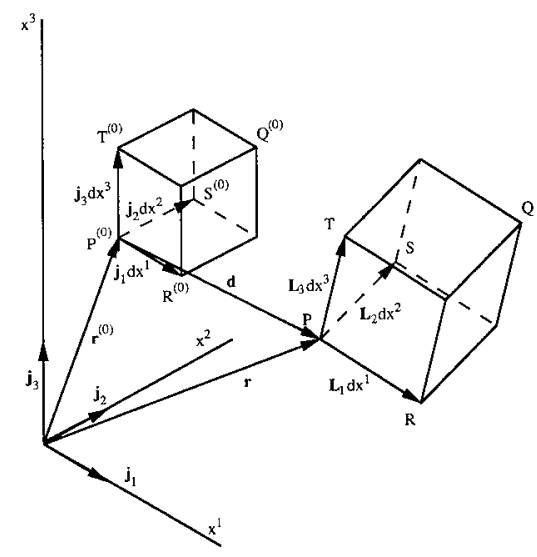

Fig. 1 Geometry of an infinitesimal parallelepiped.

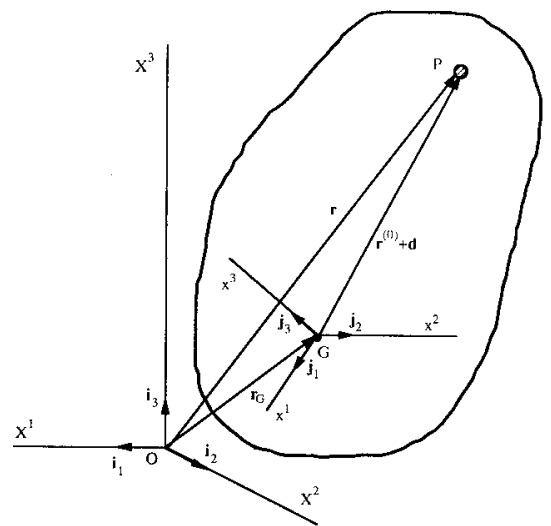

Fig. 2 Geometry of body axes and inertial axes.

In general, the lattice vectors are neither unit vectors nor are they mutually orthogonal.

Next, let us express the position vector of the point $P$ as

$$
r=r^{(0)}+d
$$

where $\boldsymbol{d}$ is the elastic displacement vector expressed in the $j$-frame. From Eqs. (4) and (5), we have

$$
\boldsymbol{L}_{\mu}=\left(\delta_{\mu}^{\chi}+d_{, \mu}^{\chi}\right) j_{\chi}
$$

where $\delta_{u}^{x}$ is the Kronecker symbol. The summation convention will be employed hereafter. Therefore a Greek letter index $\mu, \zeta, \chi$, or $\kappa$ that appears twice in the same term indicates a summation over $(1,2,3)$. Consequently, Green's strain tensor can be calculated in terms of the displacement components as follows:

$$
e_{\mu \zeta}=1 / 2\left(d_{, \zeta}^{\mu}+d_{, \mu}^{\zeta}+d_{, \mu}^{\chi} d_{, \zeta}^{\chi}\right)
$$

\section{Analysis of Stress and Stress-Strain Relations}

The force equation for the equilibrium of the deformed parallelepiped is given by $^{21}$

$$
\boldsymbol{\sigma}_{, \mu}^{\mu}+\boldsymbol{F}=\mathbf{0}
$$

where $\boldsymbol{\sigma}^{\mu}$ is the second Piola-Kirchhoff stress vector acting on the $\mu$ th face of the deformed parallelepiped, and $F$ is the body force acting in this parallelepiped. We note that $\sigma^{\mu}$ is defined per unit area, and $F$ is defined per unit volume, both with respect to the undeformed state.

Assume that the material is isotropic. The stress-strain relation is ${ }^{21}$

$$
\sigma^{\mu \zeta}=\frac{E \nu}{(1+\nu)(1-2 \nu)} e_{\kappa \kappa} \delta_{\mu \zeta}+2 G e_{\mu \zeta}
$$

where Young's modulus $E$, Poisson's ratio $\nu$, and the shear modulus $G$ are related by $E=2(1+\nu) G$.

\section{Dynamics of an Elastic Body}

Let a rectangular Cartesian coordinate system $X^{1} X^{2} X^{3}$ be fixed in inertial space, and let the corresponding unit vector system be $\boldsymbol{i}_{1} \boldsymbol{i}_{2} \boldsymbol{i}_{3}$, as shown in Fig. 2 . The origin of the bodyaxis system $x^{1} x^{2} x^{3}$ is located at a position $r_{G}$ relative to the origin of the inertial frame. Then the inertial position vector $r$ (see Fig. 2) of a representative point $P$ of the elastic body is

$$
r=r_{G}+r^{(0)}+d
$$

where we recall that $\boldsymbol{r}^{(0)}\left(x^{1}, x^{2}, x^{3}\right)$ is its position relative to the body frame before deformation, and $\boldsymbol{d}$ is its elastic deformation vector relative to the body frame. The vector $r_{G}$ is ex- 


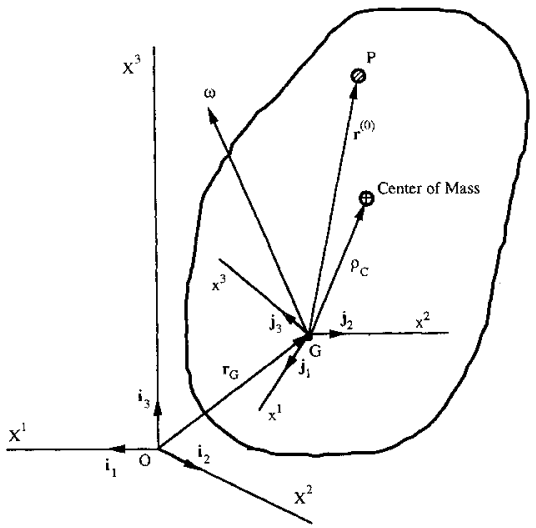

Fig. 3 Geometry of a rigid body.

pressed in terms of its inertial components, but $\boldsymbol{r}^{(0)}$ and $\boldsymbol{d}$ are expressed in $\boldsymbol{j}$-frame components; that is,

$$
\boldsymbol{r}^{(0)}=x^{\mu} \boldsymbol{j}_{\mu} \quad \text { and } \quad \boldsymbol{d}=d^{\mu} \boldsymbol{j}_{\mu}
$$

From Eq. (10), we obtain

$$
\frac{\mathrm{d} \boldsymbol{r}}{\mathrm{d} t}=\frac{\mathrm{d} \boldsymbol{r}_{G}}{\mathrm{~d} t}+\left(\frac{\mathrm{d} d}{\mathrm{~d} t}\right)_{r}+\omega \times\left(r^{(0)}+d\right)
$$

where the absolute angular velocity of the $j$-frame is

$$
\omega=P j_{1}+Q j_{2}+R j_{3}
$$

and where $(\mathrm{d} d / \mathrm{d} t)_{r}$ is the rate change of $d$ as viewed from this rotating frame in which the $\boldsymbol{j}_{\mu}$ unit vectors are constant.

The dynamical equations of motion are obtained by using d'Alembert's principle in its Lagrangian form involving virtual work. Here we lump the inertia force $-\rho\left(d^{2} r / \mathrm{d} t^{2}\right)$ with the body force $F$, each per unit volume. We consider an elastic body that is subject to body forces $F\left(x^{1}, x^{2}, x^{3}, t\right)$ distributed throughout the body, surface forces $B\left(x^{1}, x^{2}, x^{3}, t\right)$ applied on the surface $S_{1}$, and specified surface displacements $r\left(x^{1}, x^{2}, x^{3}, t\right)$ on the surface $S_{2}$. The virtual work expression for the dynamical problem is

$$
\begin{aligned}
& \iiint_{V} \boldsymbol{\sigma}^{\mu} \cdot(\delta \boldsymbol{r})_{, \mu} \mathrm{d} V-\iiint_{V}\left(\boldsymbol{F}-\rho \frac{\mathrm{d}^{2} \boldsymbol{r}}{\mathrm{d} t^{2}}\right) \cdot \delta r \mathrm{~d} V \\
& -\iint_{S_{1}} \boldsymbol{B} \cdot \delta \boldsymbol{r} \mathrm{d} S=0
\end{aligned}
$$

The first integral represents the first variation of the stored elastic energy, whereas the second integral represents the virtual work of the body forces and inertia forces, and the third integral represents the virtual work of the surface forces acting on $S_{1}$. As the motion of the surface $S_{2}$ is prescribed, it does not enter into the virtual work expression. The virtual displacement $\delta \boldsymbol{r}$ is given by

$$
\delta \boldsymbol{r}=\delta \boldsymbol{r}_{G}+\delta d^{\mu} \boldsymbol{j}_{\mu}+\delta \boldsymbol{\theta} \times\left(\boldsymbol{r}^{(0)}+\boldsymbol{d}\right)
$$

where $\delta \boldsymbol{\theta}$ is the virtual rotation of the body axes; i.e., the $j$-frame.

\section{General Dynamical Equations of Motion}

Let us begin with the matrix equation relating the unit vectors of the body-axis $j$-frame and the inertial $i$-frame, where $\boldsymbol{A}$ is an orthogonal matrix:

$$
j_{\chi}=A i_{\zeta}
$$

Using Eq. (6), we see that $L_{x}$ and $\boldsymbol{i}_{\zeta}$ are related by

$$
L_{\chi}=\boldsymbol{Z} \boldsymbol{j}_{\kappa}=\boldsymbol{Z} \boldsymbol{A} \boldsymbol{i}_{\zeta}
$$

where $Z$ is the nonorthogonal matrix:

$$
[Z]=\left[\begin{array}{ccc}
1+d_{, 1}^{1} & d_{, 1}^{2} & d_{, 1}^{3} \\
d_{, 2}^{1} & 1+d_{, 2}^{2} & d_{, 2}^{3} \\
d_{, 3}^{1} & d_{, 3}^{2} & 1+d_{, 3}^{3}
\end{array}\right]
$$

\section{General Case: Elastic Body}

We can write Eq. (14) in the following form and see each term more explicitly:

$$
\begin{aligned}
& \iiint_{V} F^{\chi} \boldsymbol{j}_{\chi} \cdot \delta r^{\zeta} \boldsymbol{i}_{\zeta} \mathrm{d} V_{i}+\iint_{S_{1}} \boldsymbol{B}^{\chi} \boldsymbol{j}_{\chi} \cdot \delta r^{\zeta} \boldsymbol{i}_{\zeta} \mathrm{d} S \\
& \quad-\iiint_{\int_{V}} \rho \frac{\mathrm{d}^{2} r^{\chi}}{\mathrm{d} t^{2}} \boldsymbol{i}_{\chi} \cdot \delta r^{\zeta} \boldsymbol{i}_{\zeta} \mathrm{d} \boldsymbol{V} \\
& \quad-\iiint_{V} \sigma^{\mu \times} \boldsymbol{L}_{\chi} \cdot\left(\delta r^{\zeta} \boldsymbol{i}_{\zeta}\right)_{, \mu} \mathrm{d} V=\mathbf{0}
\end{aligned}
$$

We can also rewrite Eq. (15) as follows:

$$
\delta r^{\zeta} \boldsymbol{i}_{\zeta}=\delta r_{G}^{\zeta} \boldsymbol{i}_{\zeta}+\delta d^{\zeta} \boldsymbol{j}_{\zeta}+\delta \Theta^{\zeta} \boldsymbol{j}_{\zeta} \times\left(r^{(0) \chi} \boldsymbol{j}_{\chi}+d^{\chi} \boldsymbol{j}_{\chi}\right)
$$

Let $\left\{\sigma_{I}^{\mu}\right\}$ be the $\boldsymbol{L}$-frame skewed components and let $\left\{\sigma_{I}^{\mu}\right\}$ be the $i$-frame components of the same stress vector $\sigma^{\mu}$. Then

$$
\left\{\sigma_{I}^{\mu}\right\}=[A]^{T}[Z]^{T}\left\{\sigma_{L}^{\mu}\right\}
$$

Now we revert to the notation $\left\{\sigma_{L}^{\mu}\right\} \equiv\left\{\sigma^{\mu}\right\}$ and write Eqs. (19) and (20) in matrix form as

$$
\begin{aligned}
& \iiint_{V}\left([A]^{T}\{F\}\right)^{T}\{\delta r\} \mathrm{d} V+\iint_{S_{1}}\left([A]^{T}\{B\}\right)^{T}\{\delta r\} \mathrm{d} S \\
& -\iiint_{V} \rho\left\{\frac{\mathrm{d}^{2} r}{\mathrm{~d} t^{2}}\right\}^{T}\{\delta r\} \mathrm{d} V \\
& -\iiint_{V}\left([A]^{T}[Z]^{T}\left\{\sigma^{\mu}\right\}\right)^{T}\left\{\delta r_{, \mu}\right\} \mathrm{d} V=0
\end{aligned}
$$

and

$$
\{\delta r\}=\left\{\delta r_{G}\right\}+[A]^{T}\{\delta d\}+[A]^{T}[\delta \tilde{\Theta}]\left(\left\{r^{(0)}\right\}+\{d\}\right)
$$

Here $[\tilde{\delta}]$ is a $3 \times 3$ skew-symmetric matrix representing the vector cross-product. In addition to the preceding two expressions, we still need to know $\left\{\delta r_{, \mu}\right\}$. Note that $\left\{r_{G}\right\}$ is not a function of body axis variables; we obtain

$$
\left\{\delta r_{, \mu}\right\}=[A]^{T}\left\{\delta d_{, \mu}\right\}+[A]^{T}[\tilde{\delta} \tilde{\Theta}]\left(\left\{r_{, \mu}^{(0)}\right\}+\left\{d_{, \mu}\right\}\right)
$$

Suppose we have $n$ generalized coordinates, and those corresponding to elastic displacements are associated with assumed deflection forms. Then, in terms of $x$ and $q$, we have

$$
\left\{r_{G}\right\}=\left\{r_{G}(q, t)\right\}, \quad\left\{r^{(0)}\right\}=\left\{r^{(0)}(x)\right\}, \quad\{d\}=\{d(x, q)\}
$$

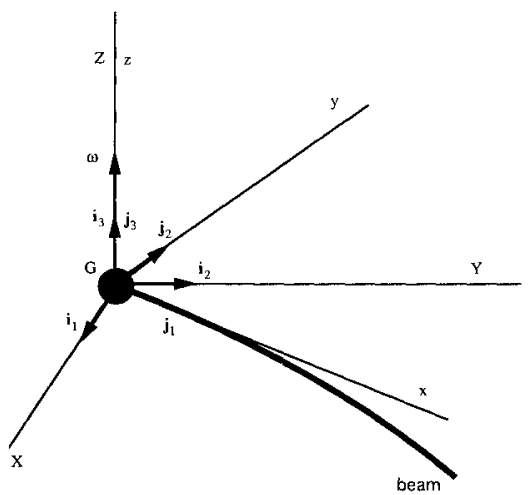

Fig. 4 Rotating cantilever beam. 
where $x$ represents the spatial variables $\left(x^{1}, x^{2}, x^{3}\right)$. The absolute velocity of the point $G$ is

$$
\left\{v_{G}\right\}=\left\{\dot{r}_{G}\right\}=\sum_{j=1}^{n} \frac{\partial}{\partial q_{j}}\left\{r_{G}\right\} \dot{q}_{j}+\frac{\partial}{\partial t}\left\{r_{G}\right\}
$$

Next, let us define the velocity coefficient $\left\{\gamma_{j}^{G}\right\}$ due to $\dot{q}_{j}$ as

$$
\left\{\gamma_{j}^{G}\right\}=\frac{\partial}{\partial \dot{q}_{j}}\left\{v_{G}\right\}=\frac{\partial}{\partial q_{j}}\left\{r_{G}\right\}
$$

where $i$-frame components are chosen. The virtual displacement $\left\{\delta r_{G}\right\}$ can be expressed as

$$
\left\{\delta r_{G}\right\}=\sum_{j=1}^{n} \frac{\partial}{\partial q_{j}}\left\{r_{G}\right\} \delta q_{j}=\sum_{j=1}^{n}\left\{\gamma_{j}^{G}\right\} \delta q_{j}
$$

The virtual displacement $\{\delta d\}$ can be derived using a similar procedure. The velocity $\{\dot{d}\}$ due to $\dot{q}_{j}$ of the generic point $P$ is a relative velocity, and we find that

$$
\{\dot{d}\}=\sum_{j=1}^{n} \frac{\partial}{\partial q_{j}}\{d\} \dot{q}_{j}
$$

The relative velocity coefficient $\left\{\gamma_{j}^{d}\right\}$ due to $\dot{q}_{j}$ is

$$
\left\{\gamma_{j}^{d}\right\}=\frac{\partial}{\partial \dot{q}_{j}}\{\dot{d}\}=\frac{\partial}{\partial q_{j}}\{d\}
$$

where the components are expressed in the $\boldsymbol{j}$-frame. So the virtual displacement $\{\delta d\}$ can be expressed as

$$
\{\delta d\}=\sum_{j=1}^{n} \frac{\partial}{\partial q_{j}}\{d\} \delta q_{j}=\sum_{j=1}^{n}\left\{\gamma_{j}^{d}\right\} \delta q_{j}
$$

and the spatial derivative of the virtual displacement $\{\delta d\}$ can also be expressed as

$$
\left\{\delta d_{, \mu}\right\}=\sum_{j=1}^{n} \frac{\partial}{\partial q_{j}}\left\{d_{, \mu}\right\} \delta q_{j}=\sum_{j=1}^{n}\left\{\gamma_{j, \mu}^{d}\right\} \delta q_{j}
$$

To obtain the virtual work due to inertial moments, we first define the angular velocity coefficient $\left\{\beta_{j}\right\}$ as follows:

$$
\left\{\beta_{j}\right\}=\frac{\partial}{\partial \dot{q}_{j}}\{\omega\}
$$

where $\{\omega\}$ is the absolute angular velocity of the $\boldsymbol{j}$-axis system. Then we can express a small virtual rotation $\{\delta \Theta\}$ in the form

$$
\{\delta \Theta\}=\sum_{j=1}^{n}\left\{\beta_{j}\right\} \delta q_{j}
$$

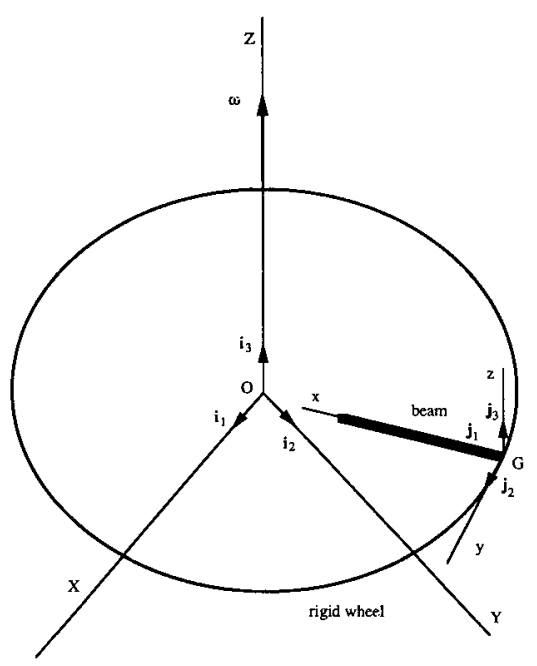

Fig. 5 Flexible beam cantilevered at the rim of a rigid wheel.
Now we are ready to consider the first integral of Eq. (22). When we substitute Eq. (23) into this integral, and use $\boldsymbol{j}$-frame components, we obtain

$$
\begin{aligned}
& \iiint_{V}\left([A]^{T}\{F\}\right)^{T}\{\delta r\} \mathrm{d} V=\iiint_{V}\{F\}^{T}\left([A]\left\{\delta r_{G}\right\}\right. \\
& \left.\quad+\{\delta d\}+[\delta \tilde{\Theta}]\left(\left\{r^{(0)}\right\}+\{d\}\right)\right) \mathrm{d} V
\end{aligned}
$$

The terms on the right-hand side of Eq. (35) represent the virtual work due to the resultant applied forces. By introducing the virtual displacements of Eqs. (28) and (31), and the virtual rotation of Eq. (34), into the right-hand side of Eq. (35), we obtain

$$
\iiint_{V}\left([A]^{T}\{F\}\right)^{T}\{\delta r\} \mathrm{d} V=\sum_{j=1}^{n} \iiint_{V}\left\{F^{B}\right\}^{T}\left\{\gamma_{j}\right\} \mathrm{d} V \delta q_{j}
$$

where the superscript $B$ denotes an applied body force. Also,

$$
\left\{\gamma_{j}\right\}=[A]\left\{\gamma_{j}^{G}\right\}+\left\{\gamma_{j}^{d}\right\}+\left[\tilde{\beta}_{j}\right]\left(\left\{r^{(0)}\right\}+\{d\}\right)
$$

and

$$
\left\{\mathcal{F}^{B}\right\}=\{\mathfrak{F}\}
$$

By a similar procedure, we obtain the second integral of Eq. (22) in the form

$$
\iint_{S_{1}}\left([A]^{T}\{B\}\right)^{T}\{\delta r\} \mathrm{d} S=\sum_{j=1}^{n} \iint_{S_{1}}\left\{F^{S}\right\}^{T}\left\{\gamma_{j}\right\} \mathrm{d} S \delta q_{j}
$$

where the superscript $S$ denotes an applied external surface force and

$$
\left\{\mathfrak{F}^{s}\right\}=\{B\}
$$

Next, let us consider the third integral of Eq. (22) where we need to compute $\{\ddot{r}\}$. We know from Eq. (10) that $r$ can be expressed in matrix form as

$$
\{r\}=\left\{r_{G}\right\}+[A]^{T}\left\{r^{(0)}\right\}+[A]^{T}\{d\}
$$

Now $[A],\left\{r_{G}\right\}$, and $\{d\}$ all vary with time, but $\left\{r^{(0)}\right\}$ does not. So, taking the derivative of Eq. (41) twice, with respect to time $t$, we obtain

$$
\begin{aligned}
\{\ddot{r}\} & =\left\{\ddot{r}_{G}\right\}+[A]^{T}[\tilde{\dot{\omega}}]\left\{r^{(0)}\right\}+[A]^{T}[\tilde{\omega}]^{2}\left\{r^{(0)}\right\} \\
+ & {[A]^{T}[\tilde{\dot{\omega}}]\{d\}+[A]^{T}[\tilde{\omega}]^{2}(d) } \\
+ & 2[A]^{T}[\tilde{\omega}]\{\dot{d}\}+[A]^{T}\{\ddot{d}\}
\end{aligned}
$$

where we note that $[\dot{A}]=-[\tilde{\omega}][A]=[\tilde{\omega}]^{T}[A]$.

Substitute Eqs. (42) and (23) into the third integral of Eq. (22), and then substitute the virtual displacements of Eqs. (28) and (31) and the virtual rotation of Eq. (34) into the former result, obtaining

$$
-\iiint_{V} \rho\left\{\frac{\mathrm{d}^{2} r}{\mathrm{~d} t^{2}}\right\}^{T}\{\delta r\} \mathrm{d} V=\sum_{j=1}^{n} \iiint_{V}\left\{F^{*}\right\}^{T}\left\{\gamma_{j}\right\} \mathrm{d} V \delta q_{j}
$$

where the asterisk denotes an inertial force. Using $j$-frame components,

$$
\begin{gathered}
\left\{\mathscr{F}^{*}\right\}=-\rho\left([A]\left\{\ddot{r}_{G}\right\}+[\tilde{\dot{\omega}}]\left\{r^{(0)}\right\}+[\tilde{\omega}]^{2}\left\{r^{(0)}\right\}\right. \\
\left.+[\tilde{\omega}]\{d\}+[\tilde{\omega}]^{2}\{d\}+2[\tilde{\omega}]\{\dot{d}\}+[\ddot{d}\}\right)
\end{gathered}
$$

Finally, we shall consider the fourth integral of Eq. (22), which involves the stress tensor. If we substitute Eq. (24) into the fourth integral of Eq. (22), and use the derivative of virtual 


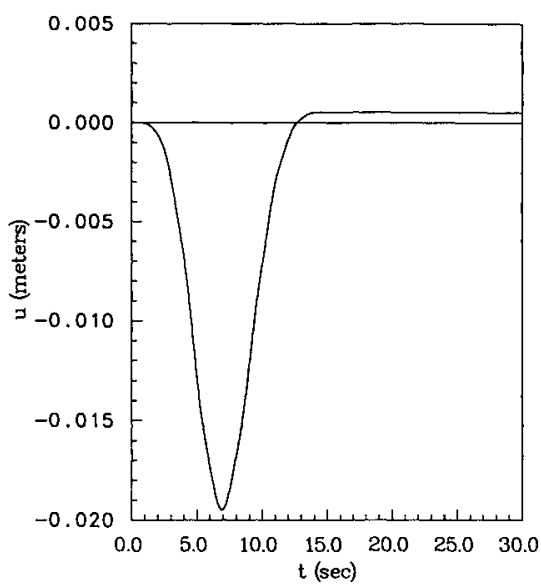

Fig. 6 The $x$ displacement $u$ vs time $t$.

displacement of Eq. (32) and of virtual rotation of Eq. (34), we obtain, after switching to $j$-frame components,

$$
\begin{aligned}
& -\iiint_{V}\left([A]^{T}[Z]^{T}\left\{\sigma^{\mu}\right\}\right)^{T}\left\{\delta r_{, \mu}\right\} \mathrm{d} V \\
& =\sum_{j=1}^{n} \iiint_{V}\left\{F^{E \mu}\right\}^{T}\left\{\gamma_{j, \mu}^{d}\right\} \mathrm{d} V \delta q_{j}
\end{aligned}
$$

where the superscript $E$ denotes an elastic force and

$$
\left\{F^{E \mu}\right\}=-[Z]^{T}\left\{\sigma^{\mu}\right\}
$$

Note that no elastic energy is stored due to a rigid body displacement, so only elastic displacements are considered here.

Next, in accordance with d'Alembert's principle, we can sum the virtual work of the applied and inertial generalized forces to obtain

$$
\begin{aligned}
& \sum_{j=1}^{n}\left(\iiint_{V}\left(\left(\left\{F^{B}\right\}+\left\{F^{*}\right\}\right)^{T}\left\{\gamma_{j}\right\}+\left\{F^{E \mu}\right\}^{T}\left\{\gamma_{j, \mu}^{d}\right\}\right) \mathrm{d} V\right. \\
& \left.\quad+\iint_{S_{1}}\left\{F^{S}\right\}^{T}\left\{\gamma_{j}\right\} \mathrm{d} S\right) \delta q_{j}=0
\end{aligned}
$$

where $\delta q$ conforms to any constraints.

Let $Q_{j}, Q_{j}^{*}$, and $Q_{j}^{E}$ be the generalized external applied forces, the generalized inertia forces, and the generalized elastic forces, respectively, and we obtain the following expressions:

$$
\begin{aligned}
Q_{j} & =\iiint_{V}\left\{F^{B}\right\}^{T}\left\{\gamma_{j}\right\} \mathrm{d} V+\iint_{S_{1}}\left\{F^{S}\right\}^{T}\left\{\gamma_{j}\right\} \mathrm{d} S \\
Q_{j}^{*} & =\iiint_{V}\left\{F^{*}\right\}^{T}\left\{\gamma_{j}\right\} \mathrm{d} V \\
Q_{j}^{E} & =\iiint_{V}\left\{F^{E \mu}\right\}^{T}\left\{\gamma_{j, \mu}^{d}\right\} \mathrm{d} V
\end{aligned}
$$

Hence, we see that d'Alembert's principle leads once again to

$$
\sum_{j=1}^{n}\left(Q_{j}+Q_{j}^{*}+Q_{j}^{E}\right) \delta q_{j}=0
$$

More explicitly, however, with the aid of Eqs. (36), (39), (43), and (45) we obtain

$$
\begin{aligned}
& \sum_{j=1}^{n}\left(Q_{j}-\iiint_{V}\left(\rho \left([A]\left\{\ddot{r}_{G}\right\}+[\tilde{\dot{\omega}}]\left\{r^{(0)}\right\}+[\tilde{\omega}]^{2}\left\{r^{(0)}\right\}\right.\right.\right. \\
& \left.\quad+[\tilde{\hat{\omega}}]\{d\}+[\tilde{\omega}]^{2}\{d\}+2[\tilde{\omega}]\{\dot{d}\}+\{\ddot{d}\}\right)^{T}\left\{\gamma_{j}\right\} \\
& \left.\left.\quad+\left([Z]^{T}\left\{\sigma^{\mu}\right\}\right)^{T}\left\{\gamma_{j, \mu}^{d}\right\}\right) \mathrm{d} V\right) \delta q_{j}=0
\end{aligned}
$$

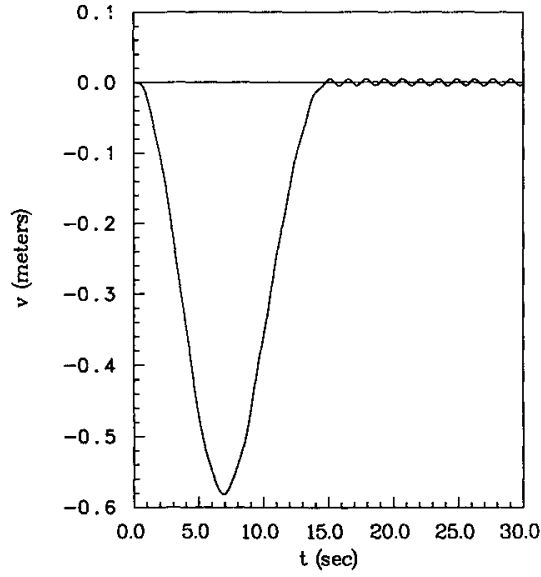

Fig. 7 The $y$ displacement $v$ vs time $t$.

Next, let us make the further assumption that the various $q$ are independent. Then each coefficient of $\delta q_{j}$ vanishes in Eq. (48) and we obtain

$$
Q_{j}+Q_{j}^{*}+Q_{j}^{E}=0, \quad(j=1,2, \ldots, n)
$$

or in greater detail,

$$
\begin{aligned}
& \iiint_{V} \rho\left([A]\left\{\ddot{r}_{G}\right\}+[\tilde{\dot{\omega}}]\left\{r^{(0)}\right\}+[\tilde{\omega}]^{2}\left\{r^{(0)}\right\}+[\tilde{\dot{\omega}}]\{d\}\right. \\
& \left.\quad+[\tilde{\omega}]^{2}\{d\}+2[\tilde{\omega}]\{\dot{d}\}+\{\ddot{d}\}\right)^{T}\left\{\gamma_{j}\right\} \mathrm{d} V \\
& \quad+\iiint_{V}\left([Z]^{T}\left\{\sigma^{\mu}\right\}\right)^{T}\left\{\gamma_{j, \mu}^{d}\right\} \mathrm{d} V=Q_{j}, \\
& (j=1,2, \ldots, n)
\end{aligned}
$$

Equation (51) is the matrix form of the general dynamical equations of motion for a system that is not subject to holonomic and nonholonomic constraints on the generalized coordinates. Now, let us take a closer look at the problems of representing the dynamical equations of constrained systems.

\section{Constrained Systems}

Consider a system whose configuration is given by $n$ generalized coordinates $q_{1}, q_{2}, \ldots, q_{n}$. Suppose there are $m$ independent constraints of the form

$$
\sum_{i=1}^{n} a_{j i}(q, t) \dot{q}_{i}+a_{j t}(q, t)=0, \quad(j=1,2, \ldots, m)
$$

where these expressions are not integrable for the case of nonholonomic constraints. If the constraints are actually holonomic and of the form

$$
\phi_{j}(q, t)=0, \quad(j=1,2, \ldots, m)
$$

then, upon differentiation with respect to time, they have the form of Eq. (52) but are integrable. In either case, let us introduce a set of $(n-m)$ independent velocity parameters $u_{j}$, known as generalized speeds, ${ }^{14}$ which are consistent with the constraints and are related to $\dot{q}$ by the equations

$$
u_{j}=\sum_{i=1}^{n} \Psi_{j i}(q, t) \dot{q}_{i}+\Psi_{j t}(q, t), \quad(j=1,2, \ldots, n-m)
$$

where $u$ may represent true velocities or quasivelocities; i.e., there is no integrability requirement on Eq. (52). If Eqs. (52) and (54) are solved for $\dot{q}$ in terms of $u$, one obtains

$$
\dot{q}_{i}=\sum_{j=1}^{n-m} \Phi_{i j}(q, t) u_{j}+\Phi_{i i}(q, t), \quad(i=1,2, \ldots, n)
$$


For this constrained system, one can use Eq. (55) to eliminate $\dot{q}$ in favor of a set of $(n-m)$ independent $u$ as velocity parameters. Then Eq. (51) still applies if one defines the velocity coefficients and angular velocity coefficients with respect to these new velocity parameters, that is,

$$
\begin{array}{ll}
\left\{\gamma_{j}^{G}\right\}=\frac{\partial}{\partial u_{j}}\left\{\dot{r}_{G}\right\}, & \left\{\gamma_{j}^{d}\right\}=\frac{\partial}{\partial u_{j}}\{\dot{d}\} \\
\left\{\gamma_{j, \mu}^{d}\right\}=\frac{\partial}{\partial u_{j}}\left\{\dot{d}_{, \mu}\right\}, & \left\{\beta_{j}\right\}=\frac{\partial}{\partial u_{j}}\{\omega\}
\end{array}
$$

Thus, we obtain $(n-m)$ dynamical equations

$$
\begin{aligned}
& \iiint_{V} \rho\left([A]\left\{\ddot{r}_{G}\right\}+[\tilde{\dot{\omega}}]\left\{r^{(0)}\right\}+[\tilde{\omega}]^{2}\left\{r^{(0)}\right\}+[\tilde{\dot{\omega}}]\{d\}\right. \\
& \left.\quad+[\tilde{\omega}]^{2}\{d\}+2[\tilde{\omega}]\{\dot{d}\}+\{\ddot{d}\}\right)^{T}\left\{\gamma_{j}\right\} \mathrm{d} V \\
& \quad+\iiint_{V}\left([Z]^{T}\left\{\sigma^{\mu}\right\}\right)^{T}\left(\gamma_{j, \mu}^{d}\right\} \mathrm{d} V=Q_{j}, \\
& (j=1,2, \ldots, n-m)
\end{aligned}
$$

where $Q$ represents generalized forces associated with $u$ and where

$$
\left\{\gamma_{j}\right\}=[A]\left\{\gamma_{j}^{G}\right\}+\left\{\gamma_{j}^{d}\right\}+\left[\tilde{\beta}_{j}\right]\left(\left\{r^{(0)}\right\}+\{d\}\right)
$$

\section{Special Case: Rigid Body}

Now, consider the special case of a rigid body motion. The origin $G$ is the reference point and we assume that this point is fixed in the body (see Fig. 3). The mass of the body is $m$, and its center mass position relative to $G$ is $\rho_{C}$. Also, we know that

$$
\iiint_{V} \rho \mathrm{d} V=m \text { and } \iiint_{V} \rho r^{(0)} \mathrm{d} V=m \rho_{C}
$$

After some algebraic manipulations, the general dynamical equation of motion (51) becomes, ${ }^{22}$ in vector form,

$$
\begin{array}{r}
m\left(\ddot{r}_{G}+\ddot{\rho}_{C}\right) \cdot \gamma_{j}^{G}+\left(\boldsymbol{I} \cdot \dot{\omega}+\boldsymbol{\omega} \times \boldsymbol{I} \cdot \boldsymbol{\omega}+m \boldsymbol{\rho}_{C} \times \ddot{r}_{G}\right) \cdot \boldsymbol{\beta}_{j}=Q_{j} \\
(j=1,2, \ldots, n)
\end{array}
$$

where there are $n$ independent generalized coordinates. Notice that the inertia dyadic $I$ is taken about its reference point at $G$.

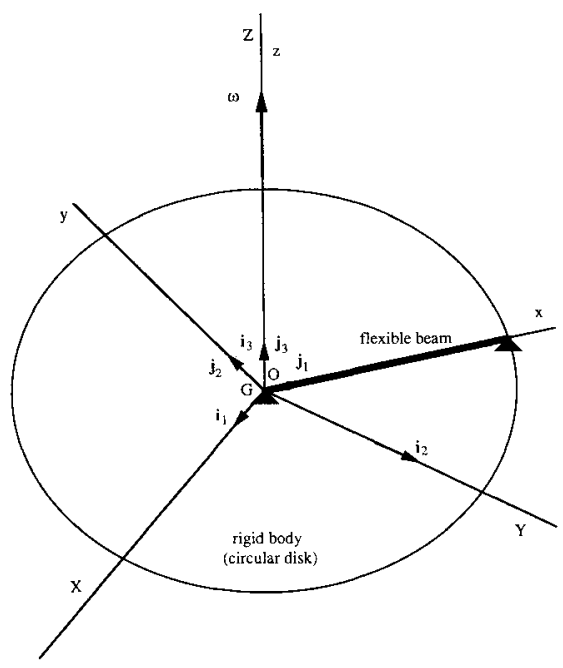

Fig. 8 Rotating pinned-pinned beam.

\section{Analysis of Flexible Beams}

When flexible structural elements are attached to a rotating base, as shown in Figs. 4 and 5, the apparent stiffness of the structural elements varies with the magnitude of the inertial angular velocity of the spinning base. For some base-element attachment configurations as in Fig. 4, the stiffness of the elements increases with base angular speed, ${ }^{23}$ whereas for others, such as Fig. 5, the stiffness decreases. ${ }^{24}$ In this section we analyze the deflection of a flexible beam with large overall motions, which performs a prescribed planar rotational motion around the $i_{3}$-axis (or $j_{3}$-axis), by using the general dynamical equations of motion.

Now let us derive the differential equations of motion for this rotating beam (see Fig. 4), whose motion is confined to the $X-Y$ plane. We choose $x y z$ as body axes and the origin $G$ as the reference point for the beam. Generalized coordinates $q_{j}$ $(j=1,2, \ldots, n)$ will be used to represent the configuration of the rotating beam at any time $t$. The velocity of the reference point $G$ is zero. This leads to the velocity coefficients $\left\{\gamma_{j}^{G}\right\}$ being equal to zero. The angular velocity of the $\boldsymbol{j}$-frame is prescribed as a function of time, so the corresponding angular velocity coefficients $\left\{\beta_{j}\right\}$ all vanish. Also, we assume that there are no applied forces, so the generalized external applied forces $Q_{j}$ are zero. But the velocity coefficients $\left\{\gamma_{j}^{d}\right\}$ resulting from elastic deformations and their spatial derivatives are nonzero.

Next, let us make the following assumptions. First, by the choice of axes and from the definition of the central line as the line of centroids of the cross sections, we have

$$
\iint_{A} y \mathrm{~d} y \mathrm{~d} z=\iint_{A} z \mathrm{~d} y \mathrm{~d} z=\iint_{A} y z \mathrm{~d} y \mathrm{~d} z=0
$$

where $A$ is the area of the cross section. Second, the centroidal displacement vector $\{D\}=\{u v w\}^{T}$ is a function of $x$ and $q$ only, and we set $w=0$. Third, we assume that the stress components $\sigma^{y y}, \sigma^{z z}, \sigma^{x z}$, and $\sigma^{y z}$ may be neglected in comparison with the other stress components, and then the stress-strain relations of interest are

$$
\sigma^{x x}=E e_{x x} \quad \text { and } \quad \sigma^{x y}=2 G e_{x y}
$$

Fourth, we employ the hypothesis that the cross sections perpendicular to the centroidal locus before bending remain plane. Also, the shape of the cross section does not change during bending.

Assume that the displacement in the $x$ direction is

$$
u(x, q)=\sum_{j=1}^{\mu_{1}} \phi_{1 j}(x) q_{1 j}
$$

where $\phi_{1 j}(x)$ is an assumed deflection form, $q_{1 j}$ is a generalized coordinate, and $\mu_{1}$ is the number of spatial functions representing $u(x, q)$. The lateral displacement $(y$ displacement), which is due to bending and shear deformations, is

$$
v(x, q)=v_{b}(x, q)+v_{s}(x, q)=\sum_{j=1}^{\mu_{2}} \phi_{2 j}(x) q_{2 j}+\sum_{j=1}^{\mu_{3}} \phi_{3 j}(x) q_{3 j}
$$

where $\phi_{2 j}(x)$ and $\phi_{3 j}(x)$ are assumed deflection forms due to bending and shear, respectively, $q_{2 j}$ and $q_{3 j}$ are the corresponding generalized coordinates, and $\mu_{2}$ and $\mu_{3}$ are the corresponding numbers of spatial functions representing bending and shear displacements $v_{b}(x, q)$ and $v_{s}(x, q)$.

We shall now consider a large deflection of an elastic beam. However, we will be satisfied to limit the problem by assuming that, although the deflection of the beam is no longer small in comparison with its height, it is still small in comparison with the longitudinal dimension of the beam. We may then employ the following expressions for total elastic displacements $d^{x}, d^{y}$, and $d^{z}$ to third order in the small quantities $y, u, v$, and $v_{b}$ :

$$
d^{x}=u-y v_{b}^{\prime}, \quad d^{y}=v-1 / 2 y\left(v_{b}^{\prime}\right)^{2}, \quad d^{z}=0
$$


where $v_{b}^{\prime}$ is the beam slope due to bending deformation, and ( )' represents $\partial() / \partial x$.

The strains $e_{\mu \nu}$ can be calculated in terms of $y, u, v$, and $v_{b}$, to third order,

$$
\begin{aligned}
& e_{x x}=u^{\prime}-y v_{b}^{\prime \prime}+1 / 2\left(u^{\prime}\right)^{2}+1 / 2\left(v^{\prime}\right)^{2}-y u^{\prime} v_{b}^{\prime \prime} \\
& e_{x y}=1 / 2\left(v^{\prime}-v_{b}^{\prime}-u^{\prime} v_{b}^{\prime}-1 / 2 v^{\prime}\left(v_{b}^{\prime}\right)^{2}\right) \\
& e_{x z}=0
\end{aligned}
$$

Since the shear deformation is small in our examples, the expression for $e_{x y}$ can be simplified to

$$
e_{x y}=1 / 2\left(v^{\prime}-v_{b}^{\prime}\right)=1 / 2 v_{s}^{\prime}
$$

which shows that the strain $e_{x y}$ is equal to one-half the beam slope due to shear deformation.

Finally, we obtain the nonlinear differential equations of motion:

\section{Numerical Simulations}

Recently it has been shown that the geometric nonlinearities arising from the coupling of longitudinal and transverse deformations have a considerable effect on the deformation of beams with large, nonsteady translational and rotational motions. ${ }^{25-32}$ In this section we simulate some rotating beam systems by using the general dynamical modeling method discussed in the previous seciton. The angular velocity $\omega$ of the spinning system as a function of time $t$ for the first two examples is

$$
\omega(t)= \begin{cases}\left(\omega_{s} / T_{s}\right)\left[t-\left(T_{s} / 2 \pi\right) \sin \left(2 \pi t / T_{s}\right)\right] j_{3} & 0 \leq t \leq T_{s} \\ \omega_{s} j_{3} & t>T_{s}\end{cases}
$$

where $T_{s}$ is the time to reach the steady-state angular velocity $\omega_{s}$. This motion is sometimes called a spin-up motion since the speed smoothly increases from 0 to $\omega_{s}$. It represents a particular example of general overall motion. The geometrical and

$$
\begin{aligned}
& \int_{L} \rho A\left\{\begin{array}{c}
-\dot{\omega} v-\omega^{2}(x+u)-2 \omega \dot{v}+\ddot{u} \\
\dot{\omega}(x+u)-\omega^{2} v+2 \omega \dot{u}+\ddot{v} \\
0
\end{array}\right\}^{T}\left\{\begin{array}{c}
\frac{\partial u}{\partial q_{j}} \\
\frac{\partial v}{\partial q_{j}} \\
0
\end{array}\right\} \mathrm{d} x+\int_{L} I_{m} \ddot{v}_{b}^{\prime} \frac{\partial v_{b}^{\prime}}{\partial q_{j}} \mathrm{~d} x+\dot{\omega} \int_{L} I_{m} \frac{\partial v_{b}^{\prime}}{\partial q_{j}} \mathrm{~d} x \\
& +\int_{L}\left\{\begin{array}{c}
E A\left(u^{\prime}+1 / 2\left(v^{\prime}\right)^{2}+3 / 2\left(u^{\prime}\right)^{2}+1 / 2 u^{\prime}\left(v^{\prime}\right)^{2}+1 / 2\left(u^{\prime}\right)^{3}\right) \\
E A\left(u^{\prime} v^{\prime}+1 / 2\left(v^{\prime}\right)^{3}+1 / 2\left(u^{\prime}\right)^{2} v^{\prime}\right) \\
0
\end{array}\right\}^{T}\left\{\begin{array}{c}
\frac{\partial u^{\prime}}{\partial q_{j}} \\
\frac{\partial v^{\prime}}{\partial q_{j}} \\
0
\end{array}\right\} \mathrm{d} x+\int_{L} E I_{z} v_{b}^{\prime \prime} \frac{\partial v_{b}^{\prime \prime}}{\partial q_{j}} \mathrm{~d} x+\int_{L} k G A v_{s}^{\prime} \frac{\partial v_{s}^{\prime}}{\partial q_{j}} \mathrm{~d} x=0
\end{aligned}
$$

where we retain terms up to third order for the first five terms, which involve $u, v_{b}$, and $v$, and to first order for the last term resulting from $v_{s}$. The terms with the rotary inertia $I_{m}=\iint_{A} \rho y^{2} \mathrm{~d} y \mathrm{~d} z$ treated as second order are included in the equations of motion during the analysis. In determining orders of magnitude, we consider area $A=\iint_{A} \mathrm{~d} y \mathrm{~d} z$ to be of zero order, and moment of inertia $I_{z}=\iint_{A} y^{2} \mathrm{~d} y \mathrm{~d} z$ to be of second order. The factor $k$ in the expression is appended to take account of the nonuniformity of the $e_{x y}$ over the cross section. numerical data used for simulation of the system are listed in Table 1.

\section{Example 1}

A cantilever beam attached to a rigid base is shown in Fig. 4. The rigid base performs a prescribed spin-up rotational motion $\omega$ [Eq. (68)] around a vertical axis, assuming $\omega_{s}=6.0$ $\mathrm{rad} / \mathrm{s}$ and beam length $L=10.0 \mathrm{~m}$. Figures 6 and 7 show the

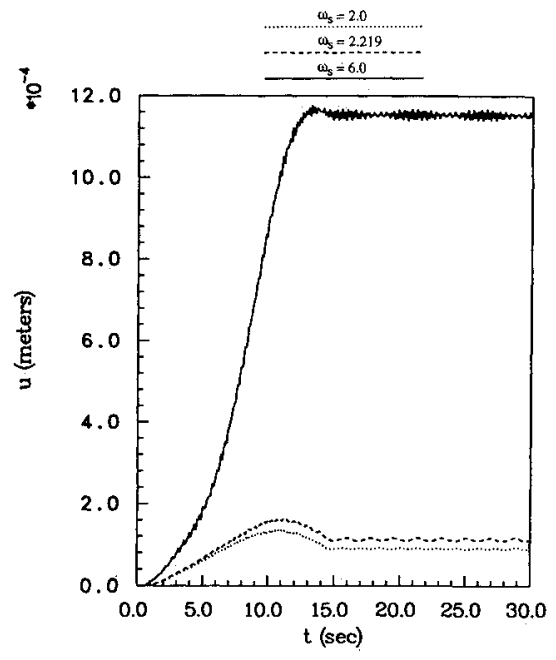

Fig. 9 Midpoint $x$ displacement $u$ vs time $t$.

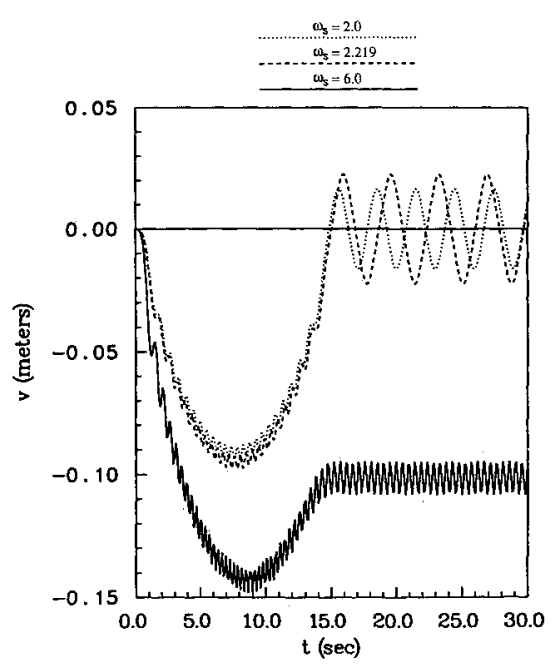

Fig. 10 Midpoint $y$ displacement $v$ vs time $t$. 
Table 1 Beam properties

\begin{tabular}{ll}
\hline \hline Density, $\mathrm{kg} / \mathrm{m}^{3}$ & $\rho=3.0 \times 10^{3}$ \\
Young's modulus, N/m $\mathrm{m}^{2}$ & $E=7.0 \times 10^{10}$ \\
Shear modulus, $\mathrm{N} / \mathrm{m}^{2}$ & $G=2.5 \times 10^{10}$ \\
Length, m & $L$ \\
Cross section area, $\mathrm{m}^{2}$ & $A=4.0 \times 10^{-4}$ \\
Area moment of inertia, $\mathrm{m}^{4}$ & $I_{z}=2.0 \times 10^{-7}$ \\
Spin-up time, s & $T_{S}=15.0$ \\
Steady-state angular velocity, rad/s & $\omega_{S}$ \\
\hline
\end{tabular}

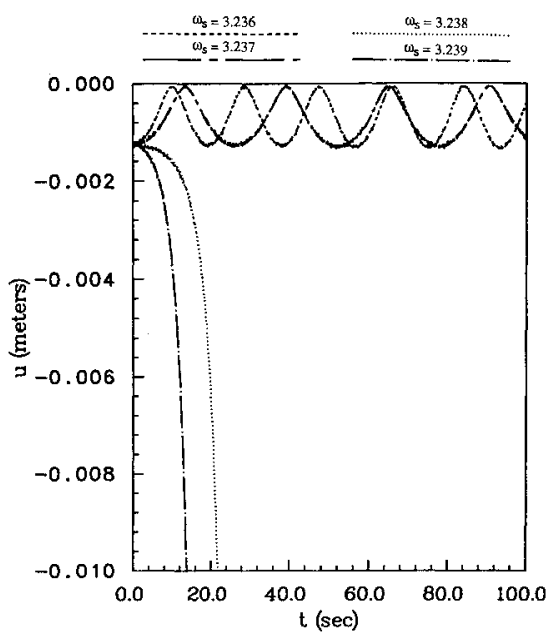

Fig. 11 The $x$ displacement $u$ vs time $t$.

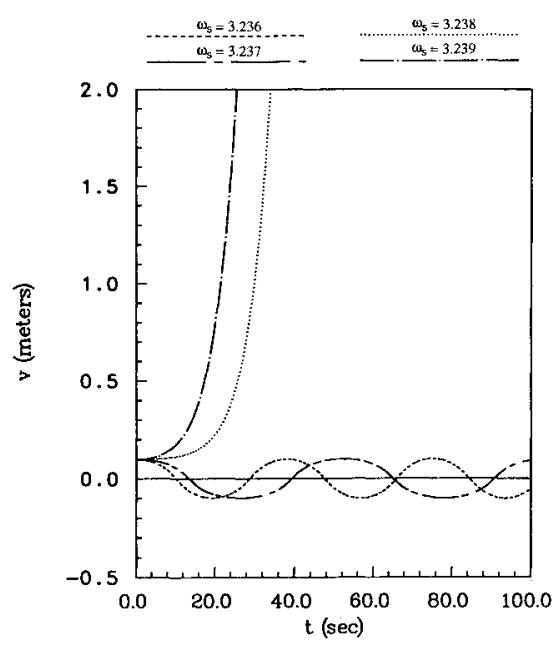

Fig. 12 The $y$ displacement $v$ vs time $t$.

plots of the $x$ displacement $u$ and the $y$ displacement $v$ of the free end of the cantilever beam.

\section{Example 2}

A beam pinned at both of its ends to a rigid body is shown in Fig. 8. The rigid body performs a rotational motion around a vertical axis passing through one end of the beam. For this system, the same type of prescribed spin-up motion is given as in Eq. (68). Three cases of final steady-state angular velocities $\omega_{s}=2.219,2.0$, and $6.0 \mathrm{rad} / \mathrm{s}$ are considered with the same beam length $L=20.0 \mathrm{~m}$. The first case gives the critical angular velocity, and a comparison of the last two cases shows the existence of residual lateral displacements if the angular velocity is larger than the critical angular velocity. Figures 9 and 10 show the plots of the $x$ displacement $u$ and the $y$ displacement $v$, respectively, of the midpoint of the pinned-pinned beam vs time $t$ for $\omega_{s}=2.219$ (dashed line), 2.0 (dotted line), and 6.0 (solid line). The physical meaning of the existence of the residual lateral displacement for $\omega_{s}=6.0$ in Fig. 10 is that the transverse centrifugal inertial force, created by the steady-state angular velocity, increases rapidly enough with $v$ to cause buckling. Nonlinear membrane forces are important in determining the final steady displacement in $v$.

\section{Example 3}

Figure 5 shows a beam cantilevered at the rim of a rigid wheel, which performs a constant rate of rotational motion around a vertical axis. By assuming the initial conditions of nonzero displacements $(q)$ and zero velocities $(\dot{q})$, the plots of the $x$ displacement $u$ and the $y$ displacement $v$ of the free end of the cantilever beam vs time $t$ for four constant angular velocities $\omega_{s}=3.236$ (dashed line), 3.237 (chain-dashed line), 3.238 (dotted line), and 3.239 (chain-dotted line) in radians/ second are shown in Figs. 11 and 12, respectively. Beam length $L=10.0 \mathrm{~m}$ is used for these simulations. When the angular speed is larger than the critical angular speed, the solution diverges. The physical meaning of the simulation result is that buckling occurs when the centrifugal inertial force, created by the steady-state angular velocity, is stronger than the elastic restoring force, and remains so with increasing deflection.

\section{Conclusions}

A general formalism has been presented, based on Lagrange's form of d'Alembert's principle and including nonlinear strain relations. This theory can be used to determine the time response of flexible structures undergoing large elastic deformations coupled with large, unsteady rotational motions. Various assumptions, such as plane sections remaining plane, are added when appropriate, but are not included in the original formulation. Several examples are included that involve the dynamics of rotating beams. The same formulation applies to cases emphasizing centrifugal stiffening, membrane effects, and buckling. No special assumptions are needed in these cases. The formulation is suitable for elastic systems with prescribed forces or displacements as functions of time, and it can be extended to the analysis of multibody systems.

\section{References}

${ }^{1}$ Hooker, W. W., and Margulies, G., "The Dynamical Attitude Equations for an $n$-Body Satellite," Journal of the Astronautical Sciences, Vol. 12, No. 4, 1965, pp. 123-128.

${ }^{2}$ Roberson, R. E., and Wittenburg, J., “A Dynamical Formalism for an Arbitrary Number of Interconnected Rigid Bodies, with Reference to the Problem of Satellite Attitude Control," Proceedings of the Third Congress of the International Federation of Automatic Control, Butterworth, London, 1967.

${ }^{3}$ Hooker, W. W., "A Set of $r$ Dynamical Attitude Equations for an Arbitrary $n$-Body Satellite Having $r$ Rotational Degrees of Freedom," AIAA Journal, Vol. 8, No. 7, 1970, pp. 1205-1207.

${ }^{4}$ Larson, V., "State Equations for an $n$-Body Spacecraft," Journal of the Astronautical Sciences, Vol. 22, No. 1, 1974, pp. 21-35.

${ }^{5}$ Likins, P. W., "Dynamic Analysis of a System of Hinge-Connected Rigid Bodies with Non-Rigid Appendages," International Journal of Solids and Structures, Vol. 9, 1973, pp. 1473-1487.

${ }^{6}$ Likins, P. W., "Finite Element Appendange Equations for Hybrid Coordinate Dynamic Analysis," International Journal of Solids and Structures, Vol. 8, 1972, pp. 709-731.

${ }^{7}$ Boland, P., Samin, J., and Willems, P., "Stability Analysis of Interconnected Deformable Bodies in a Closed-Loop Configuration," AIAA Journal, Vol. 13, No. 7, 1975, pp. 864-867.

${ }^{8}$ Hooker, W. W., "Equations of Motion for Interconected Rigid and Elastic Bodies," Celestial Mechanics, Vol. 11, No. 3, 1975, pp. 337-359.

${ }^{9}$ Hughes, P. C., "Dynamics of a Chain of Flexible Bodies," Journal of the Astronautical Sciences, Vol. 27, No. 4, 1979, pp. 359-380.

${ }^{10}$ Singh, R. P., Vandervoort, R. J., and Likins, P. W., "Dynamics of Flexible Bodies in Tree Topology-A Computer Oriented Approach," Journal of Guidance, Control, and Dynamics, Vol. 8, No. 5, 1985, pp. 584-590.

${ }^{11}$ Keat, J. E., and Turner, J. D., "Dynamic Equations of Multibody Systems with Application to Space Structure Deployment," Charles Stark Draper Lab., Rept. CSDL-T-822, Cambridge, MA, May 1983. 
${ }^{12}$ Kane, T. R., and Levinson, D. A., "Formulation of Equations of Motion for Complex Spacecraft," Journal of Guidance and Control, Vol. 3, No. 2, 1980, pp. 99-112.

${ }^{13}$ Kane, T. R., and Levinson, D. A., "The Use of Kane's Dynamical Equations in Robotics," International Journal of Robotics Research, Vol. 2, No. 3, 1983, pp. 3-21.

${ }^{14}$ Kane, T. R., Likins, P. W., and Levinson, D. A., Spacecraft Dynamics, McGraw Hill, New York, 1983.

${ }^{15}$ Huston, R. L., and Passerello, C. E., "On Lagrange's Form of d'Alembert's Principle," Matrix and Tensor Quarterly, Vol. 23, 1973, pp. 109-112.

${ }^{16}$ Greenwood, D. T., "Helicopter Rotor Dynamics," Univ. of Michigan, Dept. of Aerospace Engineering Rept., Ann Arbor, MI, Jan. 1980.

${ }^{17}$ Kaza, K. R. V., and Kvaternik, R. G., "Nonlinear Flap-Lag-Axial Functions of a Rotating Beam," AIA A Journal, Vol. 15, No. 6, 1977, pp. 871-874.

${ }^{18}$ Vigneron, F. R., "Comment on 'Mathematical Modelling of Spinning Elastic Bodies for Modal Analysis'," AIAA Journal, Vol. 13, No. 1, 1975, pp. 126-128.

${ }^{19}$ de Veubeke, B. F., "The Dynamics of Flexible Bodies," International Journal of Engineering Science, Vol. 14, 1976, pp. 895-913.

${ }^{20}$ Cavin, R. K., and Dusto, A. R., "Hamilton's Principle: Finite Element Methods and Flexible Body Dynamics," AIAA Journal, Vol. 15, No. 12, 1977, pp. 1684-1690.

${ }^{21}$ Washizu, K., Variational Methods in Elasticity and Plasticity, 3rd. ed., Pergamon, London, 1982.

${ }^{22}$ Greenwood, D. T., Principles of Dynamics, 2nd. ed., PrenticeHall, Englewood Cliffs, NJ, 1988.

${ }^{23}$ Wright, A. D., Smith, C. E., Thresher, R. W., and Wang, J. L.
C., "Vibration Modes of Centrifugally Stiffened Beams," Journal of Applied Mechanics, Vol. 49, March 1982, pp. 197-202.

${ }^{24}$ Peters, D. A., and Hodges, D. H., "In-Plane Vibration and Buckling of a Rotating Beam Clamped off the Axis of Rotation," Journal of Applied Mechanics, Vol. 47, June 1980, pp. 398-402.

${ }^{25}$ Banerjee, A. K., and Dickens, J. M., "Dynamics of an Arbitrary Flexible Body in Large Rotation and Translation," Journal of Guidance, Control, and Dynamics, Vol. 13, No. 2, 1990, pp. 221-227.

${ }^{26}$ Banerjee, A. K., and Lemak, M. E., "Multi-Flexible Body Dynamics Capturing Motion-Induced Stiffness," Journal of Applied Mechanics, Vol. 58, Sept. 1991, pp. 766-775.

${ }^{27}$ Hanagud, S., and Sarkar, S., "Problem of the Dynamics of a Cantilever Beam Attached to a Moving Base," Journal of Guidance, Control, and Dynamics, Vol. 12, No. 3, 1989, pp. 438-441.

${ }^{28}$ Ider, S. K., and Amirouche, F. M. L., "The Influence of Geometric Nonlinearities in the Dynamics and Flexible Tree-Like Structures," Journal of Guidance, Control, and Dynamics, Vol. 12, No. 6, 1989, pp. $830-837$.

${ }^{29}$ Ider, S. K., and Amirouche, F. M. L., "Nonlinear Modeling of Flexible Multibody Systems Dynamics Subjected to Variable Constraint," Journal of Applied Mechanics, Vol. 56, 1989, pp. 444-450.

${ }^{30}$ Kane, T. R., Ryan, R. R., and Banerjee, A. K., "Dynamics of a Cantilever Beam Attached to a Moving Base," Journal of Guidance, Control, and Dynamics, Vol. 10, No. 2, 1987, pp. 139-151.

${ }^{31}$ Simo, J. C., and Vu-Quoc, L., "On the Dynamics of Flexible Beams Under Large Overall Motions-The Plane Case: Part I and II," Journal of Applied Mechanics, Vol. 53, Dec. 1986, pp. 849-863.

${ }^{32}$ Christensen, E. R., and Lee, S. W., "Nonlinear Finite Element Modeling of the Dynamics of Unrestrained Flexible Structures," Computers and Structures, Vol. 23, No. 6, 1986, pp. 819-829.

Progress in Astronautics and Aeronautics Best-Selling Titles on Tactical and Strategic Missiles

\section{Tactical and Strategic Missile Guidance Paul Zarchan}

The first book to contain the guidance principles of both tactical and strategic missiles.

1990, 333 pp, illus, Hardback ISBN 0-930403-68-1

AIAA Members $\$ 50.95$

Nonmembers $\$ 65.95$

Order \#: V-124 (830)

\section{TACTICAL MISSILE SOFTWARE Paul Zarchan}

The 39 FORTRAN source code listings of Tactical and Strategic Missile Guidance, Volume 124 in the Progress in Astronautics and Aeronautics series is now available on both IBM and Macintosh formatted floppy disks. Armed with the source code listings, interested readers are better equipped to appreciate the book's concepts and explore issues beyond the scope of the text

$1991, \$ 29.95$

Order \#: PZ-Software (830)

Place your order today! Call 1-800/682-AIAA

TEST AND

EVALUATION OF THE

TACTICAL MISSILE

E.J. Eichblatt Jr., D.B. Meeker,

P.B. McQuaide, K.W. Canaga, and A. Pignataro

More than a quarter-century of experience document the trends and technologies reported in this volume. Now others in the field have the means to determine whether a missile meets its requirements, functions operationally, and should continue on into production, before a program's time and costs are scheduled, or a system is acquired.

$1989,432 \mathrm{pp}$, illus, Hardback ISBN 0-930403-56-8

AIAA Members $\$ 54.95$

Nonmembers $\$ 65.95$

Order \#: V-119 (830)
TACTICAL MISSILE AERODYNAMICS

Michael J. Hemsch and

Jack N. Nielsen, editors

This volume offers a comprehensive update of the field of tactical missile aerodynamics to aerodynamicists and designers who are actually developing future missile systems or doing research.

1986, 858 pp, illus, Hardback ISBN 0-930403-13-4

AIAA Members $\$ 69.95$

Nonmembers $\$ 99.95$

Order \#: V-104 (830)
Sales Tax: CA residents, $8.25 \% ; \mathrm{DC}, 6 \%$. For shipping and handling add $\$ 4.75$ for $1-4$ books (call for rates for higher quantities). Orders under $\$ 50.00$ must be prepaid. Please allow 4 weeks for delivery. Prices are subject to change without notice. Returns will be accepted within 15 days. 